\title{
Repetitive severe hypotension induced by indigo carmine
}

\author{
Ki Hwa Lee $\cdot$ Dong Jin Baek $\cdot$ Sang Yoon Jeon
}

Received: 22 December 2013/Accepted: 19 May 2014/Published online: 22 June 2014

(C) Japanese Society of Anesthesiologists 2014

Keywords Indigo carmine $\cdot$ Hypotension - Laparoscopic surgery

To the Editor:

Indigo carmine (IC) has been used to examine the urinary collecting system. Because of its molecular structure, it causes hypertension and bradycardia [1] and can sometimes induce hypotension.

A 66-year-old male was scheduled for robotic-assisted laparoscopic radical prostatectomy (RALP). He had a history of hypertension and showed wall motion abnormality of the right coronary artery territory. RALP proceeded under $\mathrm{CO}_{2}$ pneumoperitoneum in the $45^{\circ}$ steep Trendelenburg position. Three minutes after an intravenous (IV) injection of IC, his blood pressure (BP) decreased to $40 / 30 \mathrm{mmHg}$. Phenylephrine $100 \mu \mathrm{g}$ and crystalloid $300 \mathrm{ml}$ were administered and his BP increased. One hour after the first episode, the patient received IC again, and his BP decreased to $42 / 29 \mathrm{mmHg}$. After administration of phenylephrine and crystalloid, his BP recovered. Immunoglobulin (Ig) E was $262.0 \mathrm{IU} / \mathrm{ml}$ during the first episode and $311.0 \mathrm{IU} / \mathrm{ml}$ on the first postoperative day. The patient had no allergic histories. Hypovolemia and embolism were ruled out because of no massive bleeding and no changes in $\mathrm{SpO}_{2}$, end-tidal $\mathrm{CO}_{2}$ and peak airway pressure.

The severe hypotension was thought to have been caused by IgE-mediated histamine release. Understanding the risks of pneumoperitoneum in the steep Trendelenburg position is essential [2]. Strain on the heart due to increased venous return may precipitate heart failure in patients with compromised cardiac function. As there is limited access to patients after docking of robotic arms, special care of lines, monitors, and an endotracheal tube are necessary. It is important to know about surgical positions and to prepare for sudden and hypotensive reactions to IC, especially in cardiac-compromised patients during RALP.

Conflict of interest None.

\section{References}

1. Kawaguchi Y, Hashimoto H, Kitayama M, Hirota K. Intravenous indigo carmine might cause cerebral ischemia. Acta Anaesthesiol Scand. 2007;51:776-7.

2. Gainsburg DM. Anesthetic concerns for robotic-assisted laparoscopic radical prostatectomy. Minerva Anestesiol. 2012;78:596-604.

K. H. Lee · D. J. Baek · S. Y. Jeon $(\square)$

Department of Anesthesiology and Pain Medicine,

Haeundae Paik Hospital, College of Medicine, Inje University,

875 Haeun-daero, Haeundae-gu, Busan 612-896, Korea

e-mail: maestro@paik.ac.kr 